\title{
Addressing the needs of the telecoms industry for optical fibre communication in Africa
}

\section{Andrew Leitch, Ann Conibear}

Andrew W. R. Leitch, Ann B. Conibear, "Addressing the needs of the telecoms industry for optical fibre communication in Africa," Proc. SPIE 9664, Ninth International Topical Meeting on Education and Training in Optics and Photonics, 966421 (24 October 2005); doi: 10.1117/12.2207733 


\title{
Ref ETOP069
}

\section{Addressing the needs of the Telecoms industry for optical fibre communication in Africa}

\author{
Andrew WR Leitch and Ann B Conibear
}

\begin{abstract}
We report on a successful partnership between the Department of Physics at the Nelson Mandela Metropolitan University (NMMU) and Telkom, South Africa's national telecommunications company, to train physics students in the important fields related to optical fibre technology. The partnership, which began in 2001 and forms part of Telkom's Centre of Excellence program in South Africa, is currently being extended to other countries in Africa. The training being conducted in the Physics Department has as one of its main goals an increased understanding of polarisation mode dispersion (PMD), an effect that will ultimately limit the transmission speeds through optical fibre.
\end{abstract}

\section{Introduction}

\section{Summary}

The demand for fast and efficient communication systems in Africa is likely to increase as the pace of economic development across the continent grows. A modern system utilizing optical fibre as the backbone is required in order for a country to develop any competitive edge. Increased requirements for data transfer speeds, spurred on by the massive expansion in Internet traffic, necessitate the use of optical fibres that can handle faster bit-rates without loss of signal definition or resolution.

In South Africa, the first optical fibre links were laid more than 20 years ago - at a time when bit-rates were much slower and polarization mode dispersion (PMD) was not considered be of any consequence. The situation has radically changed today, with bit-rates of 10 gigabits per second being tested and $40 \mathrm{~Gb} / \mathrm{s}$ likely in the foreseeable future. There is therefore an urgent need to re-evaluate much of this legacy network, in order to ascertain to what extent the fibre is still suitable for the next generation network (NGN). The same applies to many other countries in Southern and Central Africa, which are increasingly looking to South Africa for leadership in economic revival.

The development of a modern optical fibre communications network requires the training of senior graduates who are familiar with the technology. For this reason Telkom SA Ltd and the Department of Physics at the Nelson Mandela Metropolitan University (NMMU) in Port Elizabeth formed a partnership in 2001 through the establishment of a Centre of Excellence (CoE) at the university, the focus being on optical fibre technologies. The broad aims of the Optical Fibre Research Group at the NMMU have been the following:

i) to train students (undergraduate as well as postgraduate) in the field of optical fibre communications;

ii) to research the various aspects of PMD; this would include having a good understanding of how to measure PMD, as well as being familiar with the various factors that may have an influence on its value;

iii) to assist Telkom in establishing the extent to which the present network is suitable for upgrade to higher bit-rates; and

iv) to be of service to the local industry, where optical fibre is cabled for various applications. 


\section{Training of undergraduates}

The training for the three-year standard BSc degree includes a geometrical optics course (given in First Year), a physical optical course (presented in Second Year) and an electromagnetic theory course (Third Year).

The Fourth Year (Honours) degree begins with an advanced electrodynamics theory course, in which basic waveguide theory is covered. The students begin to focus on specialized optical fibre technologies. They are introduced to the optical time domain reflectometry (OTDR) technique and spend some weeks learning to interpret spectra. Through experimenting with the ODTR, students become familiar with concepts such as the propagation of light through a medium, absorption and attenuation of a signal, Raleigh scattering, etc., as well as practical issues such as the use of a fusion splicer.

\section{Postgraduate training}

The two-year MSc projects form the main focus of the student training. A key part of the training is a comprehensive semester course in which the basic optical fibre technologies are covered in detail. As a result of the excellent partnership between Telkom and the research group, MSc students are able to work for periods of time with Telkom personnel, carrying out PMD and other measurements on the optical fibre network in South Africa. In this way they gain first-hand experience on making measurements in the real environment. They also gain a feel for the requirements and constraints of industry.

When considering PMD in an optical fibre, it important to realize that PMD is affected by the birefringence in the fibre. PMD has a seemingly random and complex statistical nature, which means that PMD results need to be interpreted with great care. Factors that are known to affect its value include temperature fluctuations (due to the sensitivity of the fibre core to intrinsic and extrinsic stress). In addition to birefringence, mode-coupling sites along the length of the fibre also have an influence on measured PMD, as these sites allow for the exchange of optical energy between modes.

While the interferometer technique (based on the Michelson interferometer) is widely used for PMD measurements in the field, our students also have the opportunity of using the generalized Poincaré arc technique in which the output states of polarization are determined, from which the differential group delay and hence the PMD can be found. This information is extremely valuable, especially if one is to consider compensating for the PMD in real time. The design of a PMD compensation system forms the research project for one of our PhD students.

It is worth emphasizing that the partnership with Telkom represents far more than simply a financial donation to support the activities of the research group. The association includes joint research planning and regular report-back meetings, as well as the opportunity for field measurements. An annual telecommunications conference [1] sponsored by Telkom provides an excellent platform for postgraduate students to present their results to industry and other academics.

To conclude, in addition to the support of Telkom, the Optical Fibre Research Group at the NMMU is also supported by Corning Optical Fibre (UK), Aberdare Fibre-Optic Cables (in Port Elizabeth) and Ingoma Communications Services (in Pretoria) as well as the South African government (through the NRF and Thrip). This support has enabled the research group to position itself to serve the optical fibre industry in Africa through the training of students at various levels of expertise.

[1] Satnac (Southern African Telecommunication Networks and Applications conference). http://www.satnac.org.za 